\title{
BIOSENSORS
}

\section{Janus face of DNA}

A new DNA nanodevice mediates the release of a protein from a tagged DNA aptamer in response to an arbitrary DNA molecule with no sequence complemenrity to the aptamer.

Most people immediately associate DNA with the storage of genetic information; not so physicists like Friedrich Simmel from the Center of Nanoscience at the University of Munich. Like others working in the DNA nanotechnology community, he appreciates the dual role of DNA. "What I am interested in when working with DNA is the possibility to combine simple information processing tasks with mechanical or chemical action," he explains, "you have the code of the DNA, and at the same time DNA has certain mechanical properties."

To exploit these two sides of DNA he and his team have been working with DNA aptamers, which bind other molecules in a sequence-specific manner and can be induced to release them by a conformational change. In previous work Simmel created a switchable aptamer that could be induced to release the protein thrombin upon competitive binding of a DNA strand that was complementary to the protein binding sequence of the aptamer. The limitation of this system was that it allowed no flexibility in terms of DNA input sequence, which had to be complementary to the aptamer. Simmel's goal was to uncouple the input sequence from the sequence needed for protein release.

In a recent article in Nucleic Acids Research, his team presented such a DNA device. It requires four strands of DNA and an aptamer tagged with a short sequence of choice (Fig. 1). The input strand displaces a protector strand from a connector DNA molecule, freeing it to bind the inactive, hairpin-output strand, which is complementary to the tag on the aptamer. The connector and output strands form a double-hairpin loop whose

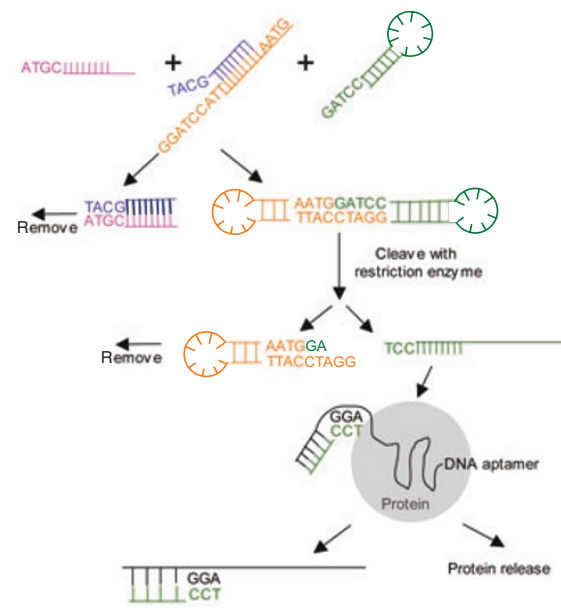

Figure 1 | Switchable DNA nanodevice. The input strand (purple) displaces the protector strand (blue) from the connector strand (orange) which then forms a double hairpin with the inactive output strand (green). Enzymatic cleavage releases the active output strand which binds to the tag sequence on the DNA aptamer (black) and leads to protein release.

\section{MIMICKING NATURE WITH PROTEIN SCAFFOLDS}

Artificial scaffolds composed of carefully chosen protein domains permit protein complexes to be assembled in vitro, providing a line of sight to construct multiprotein architectures that mimic native complexes in vivo.

In the cell, proper functioning of individual proteins often requires close association with other proteins. It is difficult, however, to examine these complex relationships in the cell because of a lack of information regarding the proteins involved, and difficulty in defining and controlling the experimental conditions. This is particularly true when it comes to the operation of macromolecular complexes. In a recent issue of Science, new work from David Tirrell's laboratory demonstrates the use of genetically encoded molecular scaffolds to provide insights into the operation of multiprotein assemblies.

Studies of molecular motors have shown that the velocities measured in vitro are often much slower than in vivo velocities, indicating that some form of cooperativity is operating in vivo, likely mediated by multiprotein complexes. Several theoretical models have attempted to explain this discrepancy and complexes have been created using nonspecific immobilization techniques, but the lack of precise control over the number of motors has been intrinsically limiting.

A scaffold capable of controlling the numerical, spatial and elastic coupling of the molecular motors would be much better suited to exploring cooperative interactions between biomotors. Michael Diehl, who headed up the project to create the scaffolds, says, "Because of my background [in molecular electronics] my first inkling was to use nanowires, [but] we realized very quickly that we were still using a nonspecific immobilization." To overcome this problem they considered engineering artificial proteins.

By using artificial proteins, "We can specify the genetic code which allows us to have residue-level control over molecular architecture, and specific and acute control over spatial, numerical and elastic coupling," remarks Diehl. Tirrell's lab is accomplished at using artificial proteins to engineer new biomaterials, and they chose to use chains of leucine zipper motifs. Each chain of one, two or three motifs linked in series forms a scaffold for high-affinity binding by biomotors linked to complementary leucine zipper motifs. 
stem is cleaved by a restriction enzyme, shortening the stem of the output strand and destabilizing its hairpin. The output strand then readily binds to the tag sequence of the aptamer and triggers protein release. In principle the input strand can be derived from any sequence, such as a gene that is overexpressed in certain disease states and would trigger the release of a therapeutic protein.

Simmel, however, cautions that such in vivo applications, though interesting, are still far from being feasible. One caveat is that not all aptamers will willingly release their bound proteins upon binding of a DNA sequence. The longer the aptamer and the more intricate its secondary structure, the more difficult it will be to trigger molecule release by competitive binding. A second problem Simmel encountered was high background; about $30 \%$ of the protein was released independently of an input signal. Simmel is confident, however, that this can be remedied by increasing the length of the output stem, thereby making it less prone to unfolding before cleavage.

Although this switchable aptamer DNA device is currently only an in vitro prototype, Simmel sees more widespread applications for this type of DNA nanotechnology: "It could become the basis of a little molecular computing unit that senses a molecule, decides something and then triggers another reaction." It may also earn its place in in vivo applications down the line, when more and more biologists take heed of the fact that DNA can do more than just store information and learn from their physicist colleagues how to harness the dual power of DNA.

\section{Nicole Rusk}

\section{RESEARCH PAPERS}

Beyer, S. \& Simmel, F.C. A modular DNA signal translator for the controlled release of a protein by an aptamer. Nucleic Acids Res. 34, 1581-1587 (2006).

Diehl knew it would be necessary to provide elasticity between the coupled molecular motors, so they inserted elastin between each leucine zipper motif in the scaffolds. He says, "We felt that elastin was going to be a very nice way to tune the elastic coupling because the viscoelastic properties of elastin are very well characterized."

The researchers chose kinesin to test the properties of their scaffold because it is a small molecular motor that has been extensively studied, and its properties are well established. Diehl et al. showed that in vitro assays using dimeric or trimeric scaffolds resulted in kinesin velocities almost twice as fast as single kinesin. By tuning the mechanical properties of the elastin domains, they also showed that coupling motors on the scaffold provided a means to reconfigure the mechanochemical mechanism of the motors.

Diehl says these scaffolds are not limited to use with molecular motors. "We can also put proteins that are coupled chemically on these scaffolds." In his lab at Rice University, Diehl is continuing to explore new uses for these scaffolds so it is almost certain that more useful applications of these scaffolds will be demonstrated shortly.

\section{Daniel Evanko}

\section{RESEARCH PAPERS}

Diehl, M.R. et al. Engineering cooperativity in biomotor-protein assemblies. Science 311, 1468-1471 (2006).

\section{NEWS IN BRIEF}

\section{IMAGING AND FLUORESCENCE}

Engineering of a monomeric green-to-red photoactivatable fluorescent protein induced by blue light

The market for photoactivatable fluorescent proteins is crowded, but Dendra offers a useful twist: sensitivity to visible blue light. Gurskaya et al. show that their engineered, monomeric variant of a coral-derived protein can undergo 1,000-4,500-fold photoconversion from green to red after exposure to $488-\mathrm{nm}$ laser light, a less cytotoxic alternative to ultraviolet light. Gurskaya, N.G. et al. Nat. Biotechnol. 24, 461-465 (2006).

\section{BIOINFORMATICS \\ Molecular dynamics simulations of the complete satellite tobacco mosaic virus}

Advances in computing power have finally allowed researchers to complete the first all-atom molecular dynamics simulation of an entire organism. Although the subject is a relatively simple RNA virus, this is a major computational achievement, and Freddolino et al. use their detailed simulation to explore important questions about the structure and assembly of the virus.

Freddolino, P.L. et al. Structure 14, 437-449 (2006).

\section{GENOMICS}

\section{Genome-wide detection of polymorphisms at nucleotide resolution with a single DNA microarray}

Working with a high-density yeast tiling array, Gresham et al. trained their SNPscanner algorithm to sensitively detect singlebase mutations throughout the genome with high resolution. With a single array experiment they were able to pinpoint individual mutations in both reference and nonreference strains, and even identify larger genomic alterations.

Gresham, D. et al. Science 311, 1932-1936 (2006).

\section{MICROBIOLOGY}

\section{Robust Salmonella metabolism limits possibilities for new antimicrobials}

Hoping to identify new genes affecting Salmonella enterica virulence, Becker et al. pooled data from previously published mouse studies, comparative genomic analyses of different S. enterica serovars, and in vivo proteomic profiling. This strategy allowed them to assemble a metabolic network map that suggests that most of the best potential drug targets may already be known.

Becker, D. et al. Nature 440, 303-307 (2006).

\section{RNA INTERFERENCE}

\section{A lentiviral RNAi library for human and mouse genes applied to an arrayed viral high-content screen}

Moffat et al. describe ongoing efforts to build a valuable research resource: a library of lentiviral constructs capable of targeting every human and mouse gene for downregulation via RNA interference. The authors test the potential of their still incomplete library in an array-based, high-content imaging screen to identify genes associated with mitotic regulation in human cancer cells.

Moffat, J. et al. Cell 124, 1283-1298 (2006). 